K. SEKIGAWA

KODAI MATH. J.

2 (i.979), 384-405

\title{
ALMOST HERMITIAN MANIFOLDS SATISFYING SOME CURVATURE CONDITIONS
}

\author{
By Kouei Sekigawa
}

\section{$\S 1$. Introduction.}

This paper is devoted to the study of almost Hermitian manifolds satisfying some curvature conditions, for example, $R(X, Y) \cdot R=0$. Let $(M, g)$ be an $m$-dimensional connected Riemannian manifold with a positive-definite metric tensor $g=\left(g_{j i}\right)$. Let $\nabla, R=\left(R_{k j i}{ }^{h}\right), R_{1}=\left(R_{j i}\right)$ and $S=g^{j i} R_{j i}$ be the Riemannian connection, the Riemannian curvature tensor, the Ricci tensor and the scalar curvature, respectively. In this paper, manifolds and tensor fields are assumed to be of class $C^{\infty}$ unless otherwise specified. In 1965, Nomizu and Yano (cf. [10]) proved the following

THEOREM 1.1. Let $g$ be an irreducible locally symmetric Riemannian metric on an m-dimensional manifold $M(m \geqq 3)$. If $g^{\prime}$ is another Riemannian metric on $M$ whose curvature tensor $R^{\prime}$ coincides with the curvature tensor $R$ of $g$, then $g^{\prime}=c g$, where $c$ is a positive constant and hence, $g^{\prime}$ is also an irreducible locally symmetruc Riemannian metric on $M$.

In a locally symmetric space $(M, g)$, at each point of $M$, we have

$$
R(X, Y) \cdot R=0, \quad \text { for all tangent vectors } X, Y,
$$

where the linear transformation $R(X, Y)$ operates on the curvature tensor $R$ as a derivation defined on the tangent tensor algebra at each point. Conversely, does the algebraic condition $\left(^{*}\right)$ on the curvature tensor $R$ imply that $\nabla R=0$ ? Nomizu gave a conjecture as follows.

CONJECTURE. Let $(M, g)$ be an m-dimensional complete, irreducible Rremannian manifold with $m \geqq 3$. If $(M, g)$ satısfies the condition $(*)$, then $(M, g)$ is locally symmetric.

Now, if the conjecture is valid, it must follow that as long as there is an irreducible and locally symmetric Riemannian metric $g$ on $M$, any metric $g^{\prime}$ on $M$ such that $R^{\prime}=R$ is also locally symmetric. This is nothing but Theorem

Received July 17, 1978 
1.1. Next, we denote by $H$ the tensor field of type $(1,5)$ on $(M, g)$ defined by $H(X, Y)=-R(X, Y) \cdot R$. If the conjecture is valid, the tensor field $H$ represents a deviation of a Riemannian metric from a locally symmetric one. However, by examples given by Takagi [19] and the present author [13], the conjecture is negative. In [14], the present author proved that the conjecture is valid in the case where $(M, g)$ is compact and irreducible, provided $\operatorname{dim} M=3$. Thus the following problem will be naturally set.

Problem. Does the algebraic condition $(*)$ on the curvature tensor of a compact and irreducible Riemannian manifold $(M, g)$ with $\operatorname{dim} M>3$ imply the fact that $(M, g)$ is locally symmetric ?

On the other hand, it might be interesting to study relations between the Riemannian structure $g$ and the almost complex structure $F$. For example, how does the Riemannian structure $g$ affect the almost complex structure $F$ in an almost Hermitian manifold $(M, F, g)$ ? In $\S 2$, we recall a theorem due to Lichnerowicz for later use. $\S 3$ will be devoted to give some formulas and theorems concerning almost Hermitian manifolds. In $\S 4$, we shall study some $K$-spaces satisfying the condition $(*)$. In $\S 5$, we shall give by using the tensor fields $H, R$ and etc., a sufficient condition for a 6 -dimensional $K$-space to be a homogeneous almost Hermitian manifold. In $\S 6$, we shall study 4-dimensional $F$-spaces and $H$-spaces satisfying the condition (*).

\section{$\S 2$. A theorem of A. Lichnerowicz.}

Let $(M, g)$ be a Riemannian manifold. Lichnerowicz [26] obtained the following formula

$$
\frac{1}{2} \Delta f=2 H^{p}{ }_{k \jmath p i h} R^{k j i h}-4\left(\nabla_{k} \nabla_{i} R_{h \jmath}\right) R^{k j i h}+\left(\nabla_{p} R_{k j i h}\right) \nabla^{p} R^{k \jmath \imath h},
$$

where $f=R_{k j \imath h} R^{k j i h}$, and $H_{p q k j i h}=-\left(\nabla_{p} \nabla_{q} R_{k j \imath h}-\nabla_{q} \nabla_{p} R_{k j \imath h}\right)$ (cf. $\S 1$ ).

In each local coordinate neighborhood, $(*)$ is equivalent to

$$
\begin{aligned}
& H_{p q k j i h}=0, \text { or } \\
& R_{p q k}{ }^{t} R_{t j \imath h}+R_{p q j}{ }^{t} R_{k t i h}+R_{p q \imath}{ }^{t} R_{k J t h}+R_{p q h}{ }^{t} R_{k j i t}=0 .
\end{aligned}
$$

From (2.1) and (2.2), Lichnerowicz proved the following

THEOREM 2.1. Let $(M, g)$ be a compact Riemannian manifold satrsfying the condition (*). If $\nabla_{k} R_{j i}=0$ holds on $M$, then $(M, g)$ is locally symmetruc.

Fujimura [3], and Sekigawa and Takagi [17] gave some generalizations of Theorem 2.1. 


\section{$\S 3$. Curvature tensors in an almost Hermitian manifold.}

Let $(M, F, g)$ be an $m(=2 n)$-dimensional almost Hermitian manifold with almost Hermitian structure $(F, g)$. If we now put

$$
R_{\jmath l}^{*}=\frac{1}{2} F_{\jmath}{ }^{t} R_{i t k}{ }^{h} F_{h}{ }^{k},
$$

then, by definition, we have

$$
F^{b a} R_{b a \jmath}{ }^{\imath}=-2 R_{t}^{* \imath} F_{\jmath}{ }^{t}, \text { and } F^{b a} R_{j b a}{ }^{\imath}=R_{t}^{* \imath} F_{\jmath}{ }^{t} .
$$

Applying the Ricci identity to $F_{\imath}{ }^{h}$, we obtain

$$
\nabla_{k} \nabla_{j} F_{\imath}{ }^{h}-\nabla_{j} \nabla_{k} F_{\imath}{ }^{h}=R_{k j t}{ }^{h} F_{\imath}{ }^{t}-R_{k j i}{ }^{t} F_{t}{ }^{h} .
$$

From (3.3), contracting with respect to $k$ and $h$, we get

$$
\nabla_{t} \nabla_{j} F_{\imath}{ }^{t}+\nabla_{j} F_{i}=\left(R_{j t}-R_{t j}^{*}\right) F_{\imath}{ }^{t},
$$

where $F_{\imath}=-\nabla_{k} F_{\imath}{ }^{k}$.

Now, operating $\nabla_{\jmath}$ to $F^{j i} F_{i h}=-\delta_{h}{ }^{3}$ gives

$$
F^{\imath} F_{i h}+F^{j i} \nabla_{j} F_{i h}=0 \text {. }
$$

Operating $\nabla^{h}=g^{h} \nabla_{2}$ to the both sides of this equation and taking account of (3.4), we have

$$
S-S^{*}=\left(\nabla^{h} F^{j i}\right) \nabla_{\jmath} F_{\imath h}-F^{\imath} F_{\imath}-2 F^{j i}\left(\nabla_{j} F_{\imath}\right),
$$

where $S^{*}=g^{j i} R_{j i}^{*}$.

Next, we shall recall the definitions of special kinds of almost Hermitian manifolds. If $(F, g)$ satisfies

$$
\nabla_{j} F_{\imath}{ }^{h}=0
$$

then $(M, F, g)$ is called a Kaehlerian space. If $(F, g)$ satisfies

$$
\nabla_{j} F_{\imath}{ }^{h}+\nabla_{i} F_{j}{ }^{h}=0,
$$

then $(M, F, g)$ is called a $K$-space (or a Tachibana space). If $(F, g)$ satisfies

$$
\nabla_{j} F_{i h}+\nabla_{i} F_{h j}+\nabla_{h} F_{j i}=0,
$$

then $(M, F, g)$ is called an $H$-space (or an almost Kaehlerian space). If $(F, g)$ satisfies

$$
\nabla_{j} \nabla_{k} F_{\imath}{ }^{h}-\nabla_{k} \nabla_{j} F_{\imath}{ }^{h}=R_{j k t}{ }^{h} F_{\imath}{ }^{t}-R_{j k \imath}{ }^{t} F_{t}{ }^{h}=0,
$$

then $(M, F, g)$ is called an $F$-space (or a para-Kaehlerian space). When $(M, F, g)$ is a Kaehlerian space, a $K$-space or an $H$-space, the condition $F_{\imath}=0$ is satisfied. 
Suppose that $(M, F, g)$ is an $H$-space. Then, by (3.8), we have

$$
\left(\nabla^{h} F^{j i}\right) \nabla_{j} F_{i h}=\frac{1}{2}\left(\nabla^{h} F^{j i}\right)\left(\nabla_{j} F_{i h}-\nabla_{i} F_{j h}\right)=-\frac{1}{2}\left(\nabla^{h} F^{j i}\right) \nabla_{h} F_{j i},
$$

and hence, by (3.5)

$$
S-S^{*}=-\frac{1}{2}\left(\nabla^{h} F^{j i}\right) \nabla_{h} F_{j i} \leqq 0 .
$$

Thus, we have the following (cf. [26])

THEOREM 3.1. In an $H$-space, we have $S \leqq S^{*}$, and the equality sign occurs if and only if the space is a Kaehlerian space.

Next, suppose the $(M, F, g)$ is a $K$-space. Then, by (3.7), we have

$$
\left(\nabla^{h} F^{j i}\right) \nabla_{j} F_{\imath h}=\left(\nabla^{h} F^{j \imath}\right) \nabla_{h} F_{\jmath \imath} \geqq 0 \text {. }
$$

Thus, we have the following (cf. [26])

THEOREM 3.2. In a $K$-space, we have $S \geqq S^{*}$, and the equality sign occurs if and only if the space is a Kaehlerian space.

We denote by $A(M, F, g)(I(M, F, g)$, resp.) the group of all automorphisms of $(M, F, g)$ (the group of all isometries of $(M, F, g)$ resp.) which acts effectively on $M$, and by $A_{0}(M, F, g)\left(I_{0}(M, F, g)\right.$, resp.) the identity component of $A(M, F, g)(I(M, F, g)$, resp.). Then, it is evident that $A(M, F, g) \subset I(M, F, g)$ (and $A_{0}(M, F, g) \subset I_{0}(M, F, g)$ ). Especially, if there exists a subgroup $G$ of $A(M, F, g)$ which acts transitively on $M$, then $(M, F, g)$ is called a homogeneous almost Hermitian manifold. Recently, concerning with the result of Ambrose and Singer [1], the present author proved the following (cf. [16])

THEOREM 3.3. Let $(M, F, g)$ be a homogeneous almost Hermutian manifold. Then, there exists a skew-symmetric tensor field $T=\left(T_{j \imath}{ }^{h}\right)$ of type $(1,2)$ on $M$ satisfying the following conditions

$$
\begin{aligned}
& \nabla_{p} R_{k j i}{ }^{h}=T_{p t}{ }^{h} R_{k j i}{ }^{t}-T_{p k}{ }^{t} R_{t j i}{ }^{h}-T_{p \jmath}{ }^{t} R_{k t \imath}{ }^{h}-T_{p \imath}{ }^{t} R_{k j t}{ }^{h}, \\
& \nabla_{p} T_{j i}{ }^{h}=T_{p t}{ }^{h} T_{j i}{ }^{t}-T_{p \jmath}{ }^{t} T_{t \imath}{ }^{h}-T_{p \imath}{ }^{t} T_{\jmath t}{ }^{h}, \\
& \nabla_{p} F_{\jmath}{ }^{h}=T_{p t}{ }^{h} F_{\jmath}{ }^{t}-T_{p \jmath}{ }^{t} F_{t}{ }^{h} .
\end{aligned}
$$

Conversely, if a complete and simply connected almost Hermitian manifold $(M, F, g)$ admits a skew-symmetric tensor field $T$ of type $(1,2)$ on $M$ satisfying the conditions $(\mathrm{A}),(\mathrm{B})$ and $(\mathrm{C})$, then $(M, F, g)$ is a homogeneous almost Hermitian manifold.

In the above Theorem, the skew-symmetricity of $T=\left(T_{j 2}{ }^{h}\right)$ means that $T_{j i h}=-T_{j h i}$ holds, where $T_{j i h}=T_{j i}{ }^{k} g_{k h}$. The rough sketch of the proof of Theorem 3.3 is as follows. Let $O(M, F, g)(U(M, F, g)$, resp.) be the ortho- 
normal frame bundle (the unitary frame bundle, resp.) over $M$ with respect to the Riemannian structure $g$ (the almost Hermitian structure $(F, g)$, resp.). We denote by $G$ the holonomy subbundle of $U(M, F, g)$ with respect to the linear connection $\nabla_{X}^{*}=\nabla_{X}-T(X)$, where $T(X) Y=T(X, Y)$. Then, $G$ acts effectively and transitively on $M$ as a group of automorphisms of $(M, F, g)$.

\section{$\S 4 . \quad K$-spaces satisf ying the condition $(*)$.}

In this section, in connection with Theorem 3.3 and the conjecture stated in $\S 1$, we shall prove the following main Theorems 4.1 and 4.2.

THEOREM 4.1. Let $(M, F, g)$ be a complete and irreducible non-Kaehlerian $K$-space satisfying the condition $\left(^{*}\right)$. Then $(M, F, g)$ is a compact and locally symmetruc space.

In this paper, when an almost Hermitian manifold $(M, F, g)$ is irreducible with respect to the Riemannian connection $\nabla$, we say that $(M, F, g)$ is a irreducible almost Hermitian manifold.

ThEOREM. 4.2. Let $(M, F, g)$ satısfy the same hypothesis as in Theorem 4.1. Assume moreover that $M$ is simply connected. Then $(M, F, g)$ is a compact and irreducible Riemannian symmetric space and furthermore, $M$ admits two actions of compact Lie groups which are effective and transitive on $M$. But these two actions are not similar to each other (cf. Remark below).

Remark. Let $G_{1}$ and $G_{2}$ be two compact, connected Lie groups which acts on a manifold $M$ effectively and transitively, $K_{1}$ and $K_{2}$ the isotropy subgroups of $G_{1}$ and $G_{2}$ respectively at some point of $M$. Denote by $\mathfrak{g}_{1}, \mathfrak{g}_{2}, \mathfrak{f}_{1}$ and $\mathfrak{f}_{2}$ the Lie algebras of $G_{1}, G_{2}, K_{1}$ and $K_{2}$, respectively. When there is an isomorphism $\phi: \mathrm{g}_{1} \rightarrow \mathrm{g}_{2}$ such that $\phi\left(\mathrm{f}_{1}\right)=\mathrm{f}_{2}$, we say that the action of $G_{1}$ is similar to that of $G_{2}$ (cf. [22]). Let $(M, g)$ be a compact and simply connected, irreducible Riemannian symmetric space, and $M=I_{0}(M, g) / K$. Let $G$ be a compact Lie group which acts on $M$ transitively and effectively. Then, it is known that, for some kinds of $\operatorname{such}(M, g)$, for example,

$$
\begin{aligned}
& S O(2 l+1) / S O(2 m) \times S O(2 l-2 m+1) \quad(2<m<l-1), \quad S O(2 l+1) / S O(2 l) \quad(l \neq 3), \\
& S O(2 l) / S O(2 m) \times S O(2 l-2 m) \quad(1<m<l-1), \quad S p(l) / S p(m) \times S p(l-m), \\
& E_{6} / S U(2) \cdot S U(6), \quad E_{7} / S U^{*}(8) \quad\left(S U^{*}(8)=S U(8) / Z_{2}\right), \quad E_{7} / S U(2) \cdot \operatorname{Sp} n(12), \\
& E_{8} / S O(16), \quad E_{8} / S U(2) \cdot E_{7}, \quad F_{4} / S U(2) \cdot S p(3), \quad F_{4} / \operatorname{Sp} n(9), \quad G_{2} / S O(4) \quad(c f . \quad[22]),
\end{aligned}
$$

the action of $G$ is always similar to the standard transitive action of $I_{0}(M, g)$ on $M$ as a Riemannian symmetric space. In [4], Fukami and Ishihara showed that there exists a Tachibana structure $(F, g)$ on a 6-dimensional sphere $S^{6}$ 
with the canonical Riemannian metric $g$ by making use of the properties of the algebra of Cayley numbers and $A_{0}\left(S^{6}, F, g\right)=G_{2}, S^{6}=G_{2} / S U(3)$. Now, let $(M, F, g)$ be a $K$-space. First of all, we shall write down some fundamental formulas in a $K$-space (cf. [9], [20], [23], etc.) as follows:

$$
F_{\jmath}{ }^{b} F_{\imath}{ }^{a} R_{b a}=R_{j i}, \quad F_{j}{ }^{b} F_{\imath}{ }^{a} R_{b a}^{*}=R_{j \imath}^{*},
$$

$$
R_{j i}^{*}=R_{\imath \jmath}^{*},
$$

$$
\left(\nabla_{j} F_{t s}\right) \nabla_{i} F^{t s}=S_{j i},
$$

$$
\left(\nabla_{j} F_{t s}\right) \nabla^{j} F^{t s}=S-S^{*} \geqq 0 \quad \text { (by (4.3) and Theorem 3.2), }
$$

$$
\nabla^{t} \nabla_{t} F_{j}^{h}=F^{h t} S_{j t},
$$

$$
R_{j i s t}-R_{b a s t} F_{j}{ }^{b} F_{\imath}{ }^{a}=-\left(\nabla_{j} F_{\imath}{ }^{r}\right) \nabla_{s} F_{t r},
$$

$$
R_{\jmath i h k}-R_{a b c d} F_{\jmath}{ }^{a} F_{\imath}{ }^{b} F_{h}{ }^{c} F_{k}{ }^{d}=0,
$$

$$
\nabla_{k} \nabla_{j} F_{\imath h}=-\frac{1}{2}\left(R_{k a i h} F_{j}{ }^{a}-R_{a k j i} F_{h}{ }^{a}+R_{a k j h} F_{\imath}{ }^{a}\right),
$$

$$
\left(R_{k j i h}-R_{k j b a} F_{\imath}{ }^{b} F_{h}{ }^{a}\right) S^{j i}=\frac{1}{4}\left(3 R_{k r}+R_{k r}^{*}\right) S_{h}{ }^{r},
$$

$$
\left(\nabla_{k} S_{j i}\right) \nabla^{k} S^{j i}=\frac{1}{8}\left(R_{b h}-5 R_{b h}^{*}\right) S^{b \imath} S_{\imath}{ }^{h},
$$

where $S_{j i}=R_{j i}-R_{j i}^{*}$.

For the sake of later use, we shall establish the following formula (4.11). Taking account of (4.3) and (4.6), we have

$$
\begin{aligned}
& R_{k j t}{ }^{v} F^{t u} R_{v u b h} F^{j b}-R_{k \jmath t}{ }^{v} F^{t u} R_{v u a^{\jmath}} F_{h}{ }^{a} \\
& =R_{k}{ }^{j}{ }^{v} F^{t u}\left(R_{v u b h} F_{\jmath}{ }^{b}+R_{v u j b} F_{h}{ }^{b}\right) \\
& =-R_{k \jmath t}{ }^{v} F^{t u} F_{h}{ }^{b}\left(\nabla^{j} F_{b}{ }^{r}\right) \nabla_{v} F_{u r} \\
& =-R_{k j t v}\left(\nabla^{v} F^{t u}\right) \nabla^{\jmath} F_{h u} \\
& =R_{k j t v}\left(R^{v t}{ }_{h}-F_{b}{ }^{v} F_{a}{ }^{t} R^{b a j}{ }_{h}\right) \\
& =\frac{1}{2}\left(R^{v t \jmath}{ }_{h}-F_{b}{ }^{v} F_{a}{ }^{t} R^{b a j}{ }_{h}\right)\left(R_{v t_{j} k}-F_{v}{ }^{q} F_{t}{ }^{p} R_{q p_{\jmath} k}\right) \\
& =\frac{1}{2}\left(\nabla^{v} F^{t r}\right)\left(\nabla^{\jmath} F_{h r}\right)\left(\nabla_{v} F_{t q}\right)\left(\nabla_{j} F_{k}{ }^{q}\right) \\
& =\frac{1}{2} S^{r q}\left(\nabla^{j} F_{h r}\right)\left(\nabla_{j} F_{k q}\right) \\
& =-\frac{1}{2} S^{r q}\left(R_{h r k q}-R_{h r b a} F_{k}{ }^{b} F_{q}{ }^{a}\right) .
\end{aligned}
$$

Thus, from the above equation and (4.9), we have 


$$
R_{k j t}{ }^{v} F^{t u} R_{v u b h} F^{j b}-R_{k j t}{ }^{v} F^{t u} R_{v u a}{ }^{j} F_{h}{ }^{a}=\frac{1}{8}\left(3 R_{h r}+R_{h r}^{*}\right) S_{k}{ }^{r} .
$$

Now, we define a tensor field $T=\left(T_{j i}{ }^{h}\right)$ of type $(1,2)$ on $M$ by

$$
T_{j i}{ }^{h}=-\frac{1}{2} F_{j}{ }^{u} \nabla_{u} F_{\imath}{ }^{h} \text {. }
$$

Then, we have

$$
T_{j i}{ }^{h}=-T_{\imath \jmath}{ }^{h}, \quad \text { and } \quad T_{j i h}=-T_{j h \imath} .
$$

By the second equation of (4.13), $T$ is skew-symmetric. We shall here prove the following

LEMmA 4.3. In a K-space, $T$ satısfles the conditıons (B) and (C) in Theorem 3.3.

Proof. From the definition of $T$ and (4.6), we have

$$
\begin{aligned}
& \nabla_{p} T_{\jmath i}{ }^{h}=\frac{1}{2}\left(R_{p j i}{ }^{h}-F_{p}{ }^{b} F_{\jmath}{ }^{a} R_{b a \imath}{ }^{h}-F_{\jmath}{ }^{t} \nabla_{p} \nabla_{t} F_{\imath}{ }^{h}\right), \\
& T_{p t}{ }^{h} T_{j i}{ }^{t}=\frac{1}{4} F_{p}{ }^{u} F_{\jmath}{ }^{v}\left(R_{u}{ }^{h}{ }_{v i}-R_{u}{ }^{h}{ }_{b a} F_{v}{ }^{b} F_{\imath}{ }^{a}\right), \\
& T_{p \jmath}{ }^{t} T_{t \imath}{ }^{h}=\frac{1}{4} F_{p}{ }^{u} F_{\jmath}{ }^{v}\left(R_{u v \imath}{ }^{h}-R_{b a \imath}{ }^{h} F_{u}{ }^{b} F_{v}{ }^{a}\right),
\end{aligned}
$$

and

$$
T_{p \imath}{ }^{t} T_{j t}{ }^{h}=\frac{1}{4} F_{p}{ }^{u} F_{\jmath}{ }^{v}\left(R_{u \imath v}{ }^{h}-R_{b a v}{ }^{h} F_{u}{ }^{b} F_{\imath}{ }^{a}\right) .
$$

From the above equations, taking account of (4.6), (4.8) and the first Bianchi identity, we have

$$
\begin{aligned}
F_{k}{ }^{\jmath} & \left(\nabla_{p} T_{j i}{ }^{h}-T_{p t}{ }^{h} T_{j i}{ }^{t}+T_{p \jmath}{ }^{t} T_{t \imath}{ }^{h}+T_{p \imath}{ }^{t} T_{\jmath t}{ }^{h}\right) \\
= & \frac{1}{2}\left(F_{k}{ }^{j} R_{p j i}{ }^{h}+F_{p}{ }^{u} R_{u k \imath}{ }^{h}+\nabla_{p} \nabla_{k} F_{\imath}{ }^{h}\right) \\
& +\frac{1}{4}\left(F_{p}{ }^{u} R_{k \imath u}{ }^{h}-F_{u}{ }^{h} R_{k \imath p}{ }^{u}-F_{p}{ }^{u} R_{u k \imath}{ }^{h}-F_{k}{ }^{u} R_{p u \imath}{ }^{h}-F_{p}{ }^{u} R_{u \imath k}{ }^{h}-F_{\imath}{ }^{u} R_{p u k}{ }^{h}\right) \\
= & \frac{1}{2} \nabla_{p} \nabla_{k} F_{\imath}{ }^{h}+\frac{1}{4}\left(F^{h u} R_{p u k i}-F_{\imath}{ }^{u} R_{p u k}{ }^{h}+F_{k}{ }^{u} R_{p u \imath}{ }^{h}\right) \\
& +\frac{1}{4} F_{p}{ }^{u}\left(R_{k \imath u}{ }^{h}+R_{u k \imath}{ }^{h}+R_{\imath u k}{ }^{h}\right) \\
= & \frac{1}{2}\left(\nabla_{p} \nabla_{k} F_{\imath}{ }^{h}+\frac{1}{2}\left(R_{p u \imath}{ }^{h} F_{k}{ }^{u}-R_{u p k i} F^{h u}+R_{u p k}{ }^{h} F_{\imath}{ }^{u}\right)\right)=0 .
\end{aligned}
$$

Thus, $T$ satisfies the condition (B). Next, using (3.7), we have

$$
F_{p}{ }^{u}\left(\nabla_{u} F_{t}{ }^{h}\right) F_{j}{ }^{t}=-F_{p}{ }^{u}\left(\nabla_{u} F_{j}{ }^{t}\right) F_{t}{ }^{h}=F_{p}{ }^{u}\left(\nabla_{j} F_{u}{ }^{t}\right) F_{t}{ }^{h}=-F_{u}{ }^{t}\left(\nabla_{j} F_{p}{ }^{u}\right) F_{t}{ }^{h}
$$




$$
\begin{aligned}
& =\nabla_{j} F_{p}{ }^{h}, \\
F_{p}{ }^{u}\left(\nabla_{u} F_{j}{ }^{t}\right) F_{t}{ }^{h} & =-F_{p}{ }^{u}\left(\nabla_{j} F_{u}{ }^{t}\right) F_{t}{ }^{h}=F_{u}{ }^{t}\left(\nabla_{j} F_{p}{ }^{u}\right) F_{t}{ }^{h}=-\nabla_{j} F_{p}{ }^{h} .
\end{aligned}
$$

Taking account of the above equations, we have

$$
\nabla_{p} F_{\jmath}{ }^{n}-T_{p t}{ }^{n} F_{\jmath}{ }^{t}+T_{p j}{ }^{t} F_{t}{ }^{n}=\nabla_{p} F_{\jmath}{ }^{h}+\frac{1}{2} \nabla_{j} F_{p}{ }^{n}+\frac{1}{2} \nabla_{j} F_{p}{ }^{h}=0 .
$$

Thus, $T$ satisfies the condition (C).

Q.E.D.

As a consequence of Lemma 4.3, we can now prove Theorem 4.4 given later. Suppose that a $K$-space $(M, F, g)$ is locally symmetric. Then, by (4.7), we have

$$
\left(\nabla_{p} F_{\jmath}{ }^{u}\right) F_{u}{ }^{t} R_{t i h k}+\left(\nabla_{p} F_{\imath}{ }^{u}\right) F_{u}{ }^{t} R_{j t h k}+\left(\nabla_{p} F_{h}{ }^{u}\right) F_{u}{ }^{t} R_{j i t k}+\left(\nabla_{p} F_{k}{ }^{u}\right) F_{u}{ }^{t} R_{j i h t}=0 .
$$

Thus, the above equation together with (3.7), (4.12) and (4.13) implies

$$
T_{p j}{ }^{t} R_{t i \hbar k}+T_{p \imath}{ }^{t} R_{j t h k}+T_{p h}{ }^{t} R_{j i t k}+T_{p k}{ }^{t} R_{j i h t}=0,
$$

which means that $T$ satisfies the condition (A). Consequently, from Theorem 3.3 and Lemma 4.3, we have the following

THEOREM 4.4. Let $(M, F, g)$ be a complete, simply connected and locally symmetric $K$-space. Then $(M, F, g)$ is a homogeneous almost Hermitıan manifold.

Proof of Theorem 4.1. Let $(M, F, g)$ be a complete and irreducible nonKaehlerian $K$-space satisfying the condition $\left(^{*}\right)$. Then the condition $\left(^{*}\right)$ implies in particular

$$
R_{k j t}{ }^{h} R_{\imath}{ }^{t}-R_{k j i}{ }^{t} R_{t}{ }^{h}=0 .
$$

By the definition of $R_{j i}^{*}$ and the condition $(*)$, we have

$$
R_{t}^{* \imath} R_{h}{ }^{t}-R_{h}^{* t} R_{t}^{2}=0 .
$$

By a straightforward calculation, we get

$$
\begin{aligned}
\nabla_{p} \nabla_{k} R_{j i}^{*}=\frac{1}{2} & \left(\left(\nabla_{p} \nabla_{k} F^{b a}\right) R_{b a t i} F_{\jmath}{ }^{t}+\left(\nabla_{k} F^{b a}\right)\left(\nabla_{p} R_{b a t \imath}\right) F_{\jmath}{ }^{t}\right. \\
& +\left(\nabla_{k} F^{b a}\right) R_{b a t \imath} \nabla_{p} F_{\jmath}{ }^{t}+\left(\nabla_{p} F^{b a}\right)\left(\nabla_{k} R_{b a t \imath}\right) F_{\jmath}{ }^{t} \\
& +F^{b a}\left(\nabla_{p} \nabla_{k} R_{b a t \imath}\right) F_{\jmath}{ }^{t}+F^{b a}\left(\nabla_{k} R_{b a t \imath}\right) \nabla_{p} F_{\jmath}{ }^{t} \\
& +\left(\nabla_{p} F^{b a}\right) R_{b a t i}\left(\nabla_{k} F_{\jmath}{ }^{t}\right)+F^{b a}\left(\nabla_{p} R_{b a t \imath}\right) \nabla_{k} F_{\jmath}{ }^{t} \\
& \left.+F^{b a} R_{b a t \imath} \nabla_{p} \nabla_{k} F_{\jmath}{ }^{t}\right) .
\end{aligned}
$$

Taking account of (3.2), (3.3) and $(*)$, we have, from the above equation

$$
\nabla_{p} \nabla_{k} R_{j i}^{*}-\nabla_{k} \nabla_{p} R_{j i}^{*}=-R_{p k j}{ }^{t} R_{t i}^{*}-R_{p k \imath}{ }^{t} R_{j t}^{*}
$$




$$
\begin{aligned}
& =R_{p k s}{ }^{b} F^{s a} R_{b a t i} F_{\jmath}{ }^{t}-R_{a i}^{*} F_{t}{ }^{a}\left(R_{p k s}{ }^{t} F_{\jmath}{ }^{s}-R_{p k j}{ }^{s} F_{s}{ }^{t}\right) \\
& =R_{p k s}{ }^{b} F^{s a} R_{b a t i} F_{\jmath}{ }^{t}-R_{a i}^{*} R_{p k s}{ }^{t} F_{t}{ }^{a} F_{\jmath}{ }^{s}-R_{a i}^{*} R_{p k \jmath}{ }^{a} .
\end{aligned}
$$

Now, by making use of the definition of $S_{j i}$, we have easily

$$
\begin{aligned}
& \left(R_{k j i h}-R_{k j b a} F_{\imath}{ }^{b} F_{h}{ }^{a}\right) S^{j \imath} \\
& \quad=R_{k j i h} R^{j i}-R_{k j i h} R^{* j i}-R_{k j b a} F_{\imath}{ }^{b} F_{h}{ }^{a} R^{j i}+R_{k j b a} F_{\imath}{ }^{b} F_{h}{ }^{a} R^{* j i} .
\end{aligned}
$$

To obtain a new formula (4.21), we shall compute the right hand side of (4.16). Taking account of the formula (**) given above, we have

$$
R_{k j i h} R^{j i}=-R_{k j h}{ }^{\imath} R_{\imath}{ }^{j}=-R_{k j \imath}{ }^{\jmath} R_{h}{ }^{\imath}=R_{k \imath} R_{h}{ }^{2} .
$$

By using (3.2), (4.1) and (**), we have

$$
\begin{aligned}
R_{k j b a} F_{\imath}{ }^{b} F_{h}{ }^{a} R^{j i} & =-R_{k j b a} F_{\imath}{ }^{j} F_{h}{ }^{a} R^{i b}=-R_{k j i}{ }^{b} R_{b}{ }^{a} F^{\imath \jmath} F_{h a} \\
& =R_{t}^{* b} F_{k}{ }^{t} R_{b}{ }^{a} F_{h a}=F_{t}^{b} R_{k}^{* t} R_{b a} F_{h}{ }^{a}=R_{k t}^{*} R_{h}{ }^{t} .
\end{aligned}
$$

Because of (3.2) and (4.15), we have

$$
\begin{aligned}
R_{k j i h} R^{* \jmath i}= & R_{k}{ }^{\jmath}{ }^{\imath} R_{i h}^{*}+R_{k}{ }^{j}{ }^{b} F^{s a} R_{b a t h} F_{\jmath}{ }^{t} \\
& -R_{a h}^{*} R_{k \jmath s}{ }^{t} F_{t}{ }^{a} F^{\jmath s}-R_{a h}^{*} R_{k}{ }^{\jmath}{ }^{a} \\
= & R_{k \jmath s}{ }^{b} F^{s a} R_{b a t h} F^{j t}+R_{a h}^{*} R_{k}^{* a} .
\end{aligned}
$$

Taking account of (3.2), (4.1) and (4.15), we have

$$
\begin{aligned}
& R_{k j b a} F_{\imath}{ }^{b} F_{h}{ }^{a} R^{* \jmath \imath}=-R_{k j b a} F_{\imath}{ }^{j} R^{* i b} F_{h}{ }^{a} \\
& \quad=-\left(R_{k j i}{ }^{b} R_{b}^{* a}+R_{k \jmath s}{ }^{b} F^{s u} R_{b u t}{ }^{a} F_{\imath}{ }^{t}-R_{u}^{* a} R_{k \jmath s}{ }^{t} F_{t}{ }^{u} F_{\imath}{ }^{s}-R_{t}^{* a} R_{k j i}{ }^{t}\right) F^{\imath \jmath} F_{h a} \\
& \quad=-R_{k}{ }^{t}{ }^{v} F^{s u} R_{v u t}{ }^{a} F_{h a}+R_{t h}^{*} R_{k}{ }^{t} .
\end{aligned}
$$

Substituting $(4.17) \sim(4.20)$ into the right hand side of (4.16), we have

$$
\begin{aligned}
& \left(R_{k j i h}-R_{k j b a} F_{\imath}{ }^{b} F_{h}{ }^{a}\right) S^{j i} \\
& \quad=S_{h t} S_{k}{ }^{t}-\left(R_{k j t}{ }^{v} F^{t u} R_{v u b h} F^{j b}-R_{k j t}{ }^{v} F^{t u} R_{v u a}{ }^{j} F_{h}{ }^{a}\right)+2 R_{t h}^{*} S_{k}{ }^{t} .
\end{aligned}
$$

This equation (4.21) together with (4.9) and (4.11) implies

$$
\begin{aligned}
& \frac{1}{4}\left(3 R_{k t}+R_{k t}^{*}\right) S_{h}{ }^{t} \\
& \quad=S_{k t} S_{h}{ }^{t}-\frac{1}{8}\left(3 R_{k t}+R_{k t}^{*}\right) S_{h}{ }^{t}+2 R_{h t}^{*} S_{k}{ }^{t} .
\end{aligned}
$$

Taking account of (4.14), we have, from (4.22)

$$
S_{h}{ }^{t}\left(R_{k t}-5 R_{k t}^{*}\right)=0
$$


Transvecting (4.23) with $S^{h k}$ and using (4.10), we have finally the formula

$$
\nabla_{k} S_{j 2}=0 \text {. }
$$

Since $(M, F, g)$ is irreducible, as a consequence of (4.24), there exists a constant $c$ such that

$$
S_{j i}=c g_{j i} .
$$

Thus, from (4.23) and (4.25), we have

$$
c\left(R_{j i}-5 R_{j i}^{*}\right)=0 .
$$

Since $(M, F, g)$ is non-Kaehlerian, we have $c \neq 0$. Thus, from (4.26), we have

$$
R_{j i}=5 R_{j i}^{*} .
$$

From (4.25) and (4.27), we have

$$
R_{j i}=\frac{5}{4} c g_{j i}, \quad R_{j i}^{*}=\frac{1}{4} c g_{j i} .
$$

Using (4.4) and (4.25), we have $c>0$. Thus, $(M, F, g)$ is an Einstein space with positive scalar curvature $S=(5 / 4) m c$. Since $(M, F, g)$ is complete, by Myers' theorem, we see that $M$ is compact, and its diameter $d(M)$ satisfies $d(M) \leqq$ $2(\sqrt{ }(m-1) / 5 c) \pi$. Consequently, because of Theorem 2.1, Theorem 4.1 is proved completely.

Q.E.D.

Proof of Theorem 4.2. We assume furthermore that $M$ is simply connected. Then, by Theorem 4.1, $(M, F, g)$ is a compact, simply connected and irreducible Riemannian symmetric space, and furthermore, by Theorem $4.4,(M, F, g)$ is a homogeneous almost Hermitian manifold. Since $(M, F, g)$ is a Riemannian symmetric space, of course, the tensor field $T_{1}=0$ of type $(1,2)$ on $M$ satisfies the conditions (A) and (B) in Theorem 3.3. Let $G_{1}$ and $G$ be the holonomy subbundles over $M$ of $O(M, F, g)$ through a point of $U(M, F, g)$ with respect to the linear connections $\nabla_{X}$ and $\nabla_{X}^{*}=\nabla_{X}-T(X)$, respectively, where $T$ is the tensor field on $M$ defined by (4.12). Then, $G_{1}(G$, resp.) acts on $M$ effectively and transitively as a group of isometries of $(M, F, g)$ (automorphisms of $(M, F, g)$, resp.). Since $M$ is compact, $A_{0}(M, F, g)$ and $I_{0}(M, F, g)$ are both compact Lie groups (cf. [7]). Now, we assume that $A_{0}(M, F, g)=I_{0}(M, F, g)$ holds. Then, $G_{1} \subset A_{0}(M, F, g)$ and hence, $G_{1} \subset U(M, F, g)$. However, since $(M, F, g)$ is nonKaehlerian, this is a contradiction. Thus, $A_{0}(M, F, g)$ is a proper subgroup of $I_{0}(M, F, g)$. Thus, $\operatorname{dim} A_{0}(M, F, g)<\operatorname{dim} I_{0}(M, F, g)$ holds, and hence, the actions of $A_{0}(M, F, g)$ and $I_{0}(M, F, g)$ on $M$ are not similar to each other. Consequently, we have Theorem 4.2 .

Q.E. D.

Recently, Ogawa [11] proved the following

THEOREM 4.5. Let $(M, F, g)$ be a compact Kaehlernan space satısfynng the 
condition (*). If the scalar curvature of $(M, F, g)$ is constant, then $(M, F, g)$ is locally symmetric.

From Theorems 4.1 and 4.5 , in connection with the problem stated in $\S 1$, we have immediately the following

COROLlary 4.6. Let $(M, F, g)$ be a compact and irreducible $K$-space satısfying the condition (*). If the scalar curvature of $(M, F, g)$ is constant, then $(M, F, g)$ is locally symmetric.

\section{$\S 5$. 6-dimensional $K$-spaces.}

Let $(M, F, g)$ be a 6-dimensional complete non-Kaehlerian $K$-space. Then, besides the formulas (4.1) (4.10), the following identities hold (cf. [9], [20]).

$$
\begin{aligned}
\nabla_{k} \nabla_{j} F_{\imath h} & =-\frac{S}{30}\left(g_{k j} F_{\imath h}+g_{k \imath} F_{h j}+g_{k h} F_{j i}\right), \\
\left(\nabla_{t} F_{k j}\right) \nabla^{t} F_{i h} & =F_{\imath}{ }^{t} F_{h}{ }^{s} R_{k j t s}-R_{k j i h} \\
& =-\frac{S}{30}\left(g_{j i} g_{k h}-g_{k \imath} g_{j h}-F_{j i} F_{k h}+F_{k i} F_{j h}\right) .
\end{aligned}
$$

From (5.2), we have

$$
F_{p}{ }^{t} R_{k j t h}=-F_{h}{ }^{t} R_{k j p t}+\frac{S}{30}\left(F_{h k} g_{\jmath p}-F_{h j} g_{k p}-g_{h k} F_{\jmath p}+g_{h j} F_{k p}\right) .
$$

Transvecting (5.2) with $g^{k h}$, we get

$$
R_{j i}-R_{j i}^{*}=\frac{2 S}{15} g_{j i}
$$

from which and (4.9), taking account of Theorem 3.2,

$$
R_{j i}-5 R_{j i}^{*}=0 \text {. }
$$

From the above equations, we have

$$
R_{j i}=\frac{S}{6} g_{j i}, \quad R_{j i}^{*}=\frac{S}{30} g_{j i} .
$$

From (3.2) and (5.4), we have

$$
F^{b a} R_{b a j i}=-\frac{S}{15} F_{j i}, \quad F^{b a} R_{j b a \imath}=\frac{S}{30} F_{j i} .
$$

Now, we shall establish the integral formula (5.26) given later, which implies the following

THEOREM 5.1. Let $(M, F, g)$ be a 6-dimensional complete and simply connected non-Kaehlerian $K$-space satisfying the condition

$$
2 H_{k j p i n}^{p} R^{k j i n}+\frac{S}{15}\left(R_{k j i h} R^{k j i n}-\frac{S^{2}}{15}\right) \geqq 0 .
$$


Then, $(M, F, g)$ is a homogeneous almost Hermitıan manifold.

For the sake of later use, we shall prepare the following formulas (5.6) (5.9), (5.11) and (5.13). By making use of (5.2) (5.5), we have

$$
\begin{aligned}
R^{\jmath p i n} F_{\imath}{ }^{k} F_{p}{ }^{t} R_{k \jmath t h}=-F_{\imath}{ }^{k} F_{h}{ }^{t} R^{\jmath p i n} R_{k \jmath p t} \\
\quad+\frac{S}{30} R^{\jmath \imath \imath h} F_{\imath}{ }^{k}\left(F_{h k} g_{\jmath p}-F_{h \jmath} g_{k p}-g_{h k} F_{\jmath p}+g_{h j} F_{k p}\right) \\
=-\left(R^{k t \jmath p}-\frac{S}{30}\left(g^{j t} g^{p k}-g^{j k} g^{p t}-F^{\jmath t} F^{p k}+F^{j k} F^{p t}\right)\right) R_{k \jmath p t}-\frac{2}{75} S^{2} \\
=-R^{k t \jmath p} R_{k \jmath p t}-\frac{4}{75} S^{2} .
\end{aligned}
$$

The formulas (5.4) and (5.5) imply

$$
R^{j p i n} F_{p i} F^{k t} R_{k j t h}=-\left(\frac{S}{30}\right)^{2} F^{j h} F_{j h}=-\frac{S^{2}}{150} .
$$

Now, making use of (5.2) (5.5) and taking account of (5.6), we have

$$
\begin{aligned}
R^{\jmath p i n} F_{p}{ }^{k} F_{\imath}{ }^{t} R_{k \jmath t h}= & -F_{p}{ }^{k} F_{\imath}{ }^{t} R^{\jmath p i n} R_{k t h \jmath}-F_{p}{ }^{k} F_{\imath}{ }^{t} R^{\jmath p i n} R_{k h \jmath t} \\
= & -R^{\jmath p i n}\left(R_{p i h j}-\frac{S}{30}\left(g_{p \jmath} g_{i n}-g_{p h} g_{\imath \jmath}-F_{p j} F_{i h}+F_{p h} F_{\imath \jmath}\right)\right) \\
& -R^{k t \jmath p} R_{k \jmath p t}-\frac{4}{15} S^{2} \\
= & -R^{\jmath p i n} R_{p \imath h \jmath}+\frac{2}{75} S^{2}-R^{k t \jmath p} R_{k \jmath p t}-\frac{4}{75} S^{2} \\
= & -\frac{2}{75} S^{2} .
\end{aligned}
$$

By making use of (5.2), (5.4) and (5.5), we find

$$
\begin{aligned}
& R_{v}^{k}{ }_{v}^{i n} F_{k}{ }^{\jmath} F^{u v} R_{u j i h} \\
& \quad=-R^{k v i n}\left(R_{v k i h}-\frac{S}{30}\left(g_{k \imath} g_{v h}-g_{v \imath} g_{k h}-F_{k \imath} F_{v h}+F_{v i} F_{k h}\right)\right) \\
& \quad=R_{k \jmath i h} R^{k j i h}-\frac{4}{75} S^{2} .
\end{aligned}
$$

Furthermore, from (5.2) and (5.4), we have

$$
\begin{aligned}
& R^{\jmath p i h}\left(\nabla^{k} F_{p}{ }^{u}\right)\left(\nabla_{i} F_{u}{ }^{t}\right) R_{k j t h} \\
& \quad=-\frac{S}{30}\left(R^{\jmath p i n} R_{\imath \jmath p h}+\frac{S^{2}}{6}+R^{\jmath p i n} F_{\imath}{ }^{k} F_{p}{ }^{t} R_{k j t h}+R^{\jmath p \imath h} F_{p \imath} F^{k t} R_{k \jmath t h}\right) .
\end{aligned}
$$

Substituting (5.6) and (5.7) respectively into the third term and the forth term in the parenthesis of the right hand side of (5.10), we have 


$$
R^{\text {ppin }}\left(\nabla^{k} F_{p}{ }^{u}\right)\left(\nabla_{i} F_{u}{ }^{t}\right) R_{k j t h}=-\frac{4}{5 \cdot 15^{2}} S^{3} .
$$

From (5.1) and (5.4), we have

$$
\begin{aligned}
& R^{\jmath p \imath \hbar} F_{p}{ }^{a}\left(\nabla^{k} \nabla_{i} F_{a}{ }^{t}\right) R_{k \jmath t h} \\
& \quad=\frac{S}{30}\left(R^{\jmath p i n} R_{\imath \jmath p h}+F_{p}{ }^{k} F_{\imath}{ }^{t} R^{\jmath p i n} R_{k j t h}+\frac{S^{2}}{6}\right) .
\end{aligned}
$$

Substituting (5.8) into the second term in the parenthesis of the right hand side of (5.12), we have

$$
R^{\jmath p i n} F_{p}{ }^{a}\left(\nabla^{k} \nabla_{i} F_{a}{ }^{t}\right) R_{k j t h}=\frac{S}{30}\left(R^{\jmath p i n} R_{\imath \jmath p h}+\frac{7}{50} S^{2}\right) .
$$

To obtain the integral formula (5.26), we define a tensor field $L=\left(L_{p_{k j i h}}\right)$ by

$$
L_{p k j i h}=\nabla_{p} R_{k j i \hbar}+T_{p k}{ }^{t} R_{t j \imath h}+T_{p j}{ }^{t} R_{k t i h}+T_{p \imath}{ }^{t} R_{k j t h}+T_{p h}{ }^{t} R_{k j i t},
$$

where $T=\left(T_{j i}{ }^{h}\right)$ is the tensor field defined by (4.12). We here compute the square of the length $\|L\|$ of the tensor field $L$, that is,

$$
\begin{aligned}
& \|L\|^{2}=L_{p k j i h} L^{p k j i n} \\
& =\left(\nabla_{p} R_{k j i h}\right) \nabla^{p} R^{k j i \hbar} \\
& +2\left(\nabla^{p} R^{k j i h}\right)\left(T_{p k}{ }^{t} R_{t j i h}+T_{p \jmath}{ }^{t} R_{k t i h}+T_{p \imath}{ }^{t} R_{k j t h}+T_{p h}{ }^{t} R_{k j i t}\right) \\
& +T^{p k u} R_{u}^{j i \hbar} T_{p k}{ }^{v} R_{v j i h}+T^{p j u} R^{k}{ }_{u}^{i n} T_{p j}{ }^{v} R_{k v i h} \\
& +T^{p \imath u} R^{k \jmath}{ }_{u}^{h} T_{p \imath}{ }^{v} R_{k \jmath v h}+T^{p h u} R^{k j i}{ }_{u} T_{p h}{ }^{v} R_{k \jmath v h} \\
& +2\left(T^{p k u} R_{u}{ }^{j i h} T_{p \jmath}{ }^{v} R_{k v i h}+T^{p k u} R_{u}^{j i h} T_{p \imath}{ }^{v} R_{k \jmath v h}\right. \\
& +T^{p k u} R_{u}^{j i \hbar} T_{p h}{ }^{v} R_{k j i v}+T^{p \jmath u} R^{k}{ }_{u}^{i h} T_{p \imath}{ }^{v} R_{k \jmath v h} \\
& \left.+T^{p j u} R^{k}{ }^{i n} T_{p h}{ }^{v} R_{k j i v}+T^{p \imath u} R^{k \jmath}{ }_{u}{ }^{h} T_{p h}{ }^{v} R_{k j i v}\right) .
\end{aligned}
$$

Thus we have

$$
\begin{aligned}
\|L\|^{2}= & \left(\nabla_{p} R_{k j i h}\right) \nabla^{p} R^{k j i h}+8\left(\nabla^{p} R^{k j i h}\right) T_{p \imath}{ }^{t} R_{k j t h} \\
& +4 T_{p k}{ }^{u} R_{u j i h} T^{p k v} R_{v}{ }^{j i h} \\
& +4\left(T^{p k u} R_{u}^{j i h} T_{p \jmath}{ }^{v} R_{k v i h}+2 T^{p k u} R_{u}{ }^{j i h} T_{p \imath}{ }^{v} R_{k \jmath v h}\right) .
\end{aligned}
$$

We now compute each of four terms appearing in the expression (5.15) above. In the first step, we compute the second term of (5.15). From the definition of $T$ and the second Bianchi identity, we have

$$
\left(\nabla^{p} R^{k j i h}\right) T_{p \imath}{ }^{t} R_{k j t h}=\frac{1}{2}\left(\nabla^{p} R^{k j i h}\right)\left(F_{p}{ }^{u} \nabla_{i} F_{u}{ }^{t}\right) R_{k j t h}
$$




$$
\begin{aligned}
= & -\frac{1}{2}\left(\nabla^{k} R^{\jmath p i n}\right)\left(F_{p}{ }^{u} \nabla_{i} F_{u}{ }^{t}\right) R_{k j t h}-\frac{1}{2}\left(\nabla^{\jmath} R^{p k \imath h}\right)\left(F_{p}{ }^{u} \nabla_{i} F_{u}{ }^{t}\right) R_{k \jmath t h} \\
= & -\nabla^{k}\left(R^{\jmath p i n}\left(F_{p}{ }^{u} \nabla_{i} F_{u}{ }^{t}\right) R_{k \jmath t h}\right) \\
& +R^{\jmath p i n}\left(\nabla^{k} F_{p}{ }^{u}\right)\left(\nabla_{i} F_{u}{ }^{t}\right) R_{k j t h}+R^{\jmath p \imath h} F_{p}{ }^{u}\left(\nabla^{k} \nabla_{i} F_{u}{ }^{t}\right) R_{k \jmath t h} .
\end{aligned}
$$

Substituting (5.11) and (5.13) into the second term and the third term of the right hand side of (5.16) respectively, we have

$$
\begin{aligned}
& \left(\nabla^{p} R^{k j i h}\right) T_{p \imath}{ }^{t} R_{k \jmath t h} \\
& \quad=-\nabla^{k}\left(R^{\jmath p i \hbar}\left(F_{p}{ }^{u} \nabla_{i} F_{u}{ }^{t}\right) R_{k j t h}\right)-\frac{S}{60}\left(R_{k j i h} R^{k j i h}-\frac{S^{2}}{15}\right) .
\end{aligned}
$$

In the second step, we compute the third term in the right hand side of (5.15). By making use of (4.3) and (5.4), we have

$$
\begin{aligned}
& T_{p k}{ }^{u} R_{u j i h} T^{p k v} R_{v}^{j i h} \\
& \quad=\frac{1}{4}\left(F_{p}{ }^{a} \nabla_{k} F_{a}{ }^{u}\right)\left(F^{p b} \nabla^{k} F_{b}^{v}\right) R_{u j i h} R_{v}^{j i h} \\
& =\frac{1}{4}\left(\nabla_{k} F_{a}{ }^{u}\right)\left(\nabla^{k} F^{a v}\right) R_{u j i h} R_{v}^{j i h} \\
& =\frac{S}{30} R_{k j i h} R^{k j i h} .
\end{aligned}
$$

In the third step, we compute the forth term of the right hand side of (5.15). By making use of (5.2) and (5.5), we have

$$
\begin{aligned}
& T_{p k}{ }^{u} R_{u j i h} T^{p j v} R_{v}^{k}{ }_{v}^{i h} \\
& \quad=\frac{1}{4}\left(F_{p}^{a} \nabla_{k} F_{a}^{u}\right)\left(F^{p b} \nabla^{j} F_{b}^{v}\right) R_{u j i h} R_{v}^{k}{ }^{i h} \\
& \quad=-\frac{S}{120}\left(R_{k j i h} R^{k j i h}-\frac{2}{75} S^{2}+F_{k}{ }^{j} F^{u v} R_{v}^{k}{ }_{v}^{i h} R_{u j i h}\right) .
\end{aligned}
$$

Substituting (5.9) into the last term in the parenthesis of the right hand side of (5.19), we have

$$
T_{p k}^{u} R_{u j i h} T^{p j v} R_{v}^{k}{ }^{i h}=-\frac{S}{60}\left(R_{k j i h} R^{k j i n}-\frac{S^{2}}{25}\right) .
$$

Similarly, by making use of (5.2), (5.4) and (5.5), we have

$$
\begin{aligned}
& T_{p k}^{u} R_{u j i h} T^{p i v} R_{v}^{k j} \\
& \quad=\frac{1}{4}\left(F_{p}^{a} \nabla_{k} F_{a}^{u}\right)\left(F^{p b} \nabla^{i} F_{b}^{v}\right) R_{u j i h} R_{v}^{k J}{ }^{h} \\
& \quad=\frac{1}{4}\left(\nabla_{a} F_{k}{ }^{u}\right)\left(\nabla^{a} F^{i v}\right) R_{u j i h} R_{v}^{k J}{ }^{h}
\end{aligned}
$$




$$
=-\frac{S}{120}\left(\frac{4}{25} S^{2}-R_{u j i h} R^{\imath \jmath u h}+R_{u j \imath h} F^{u v} F_{k}{ }^{2} R_{v}^{k \jmath}{ }^{h}\right) .
$$

Substituting (5.6) into the last term in the parenthesis of the right hand side of (5.21), we have

$$
T_{p k}^{u} R_{u \jmath i h} T^{p \imath v} R_{v}^{k \jmath}=-\frac{1}{5 \cdot 15^{2}} S^{3} .
$$

Finally, substituting (5.17), (5.18), (5.20) and (5.22) into the right hand side of (5.15), we have

$$
\begin{aligned}
\|L\|^{2}= & \left(\nabla_{p} R_{k j i h}\right) \nabla^{p} R^{k j i h}-8 \nabla^{k}\left(R^{j p i n}\left(F_{p}{ }^{u} \nabla_{i} F_{u}{ }^{t}\right) R_{k j t h}\right) \\
& -\frac{2 S}{15}\left(R_{k j i n} R^{k j i n}-\frac{S^{2}}{15}\right)+\frac{2 S}{15} R_{k j i h} R^{k j i h} \\
& -\frac{S}{15}\left(R_{k j i h} R^{k j i h}-\frac{S^{2}}{25}\right)-\frac{8 S^{3}}{5 \cdot 15^{2}} \\
= & \left(\nabla_{p} R_{k j i h}\right) \nabla^{p} R^{k j i h}-8 \nabla^{k}\left(R^{\jmath p 2 h}\left(F_{p}{ }^{u} \nabla_{i} F_{u}{ }^{t}\right) R_{k j t h}\right) \\
& -\frac{S}{15}\left(R_{k j i h} R^{k j i h}-\frac{S^{2}}{15}\right) .
\end{aligned}
$$

On the other hand, because of (4.4) and (5.4), $(M, F, g)$ is an Einstein space with positive scalar curvature. Thus, by Myers' theorem, $M$ is compact, and the formula (2.1) implies in particular

$$
\left(\nabla_{p} R_{k j i h}\right) \nabla^{p} R^{k j i h}=\frac{1}{2} \Delta\left(R_{k j i h} R^{k j i h}\right)-2 H_{k j p i h}^{p} R^{k j i h} .
$$

Substituting (5.24) into (5.23), we have

$$
\begin{aligned}
\|L\|^{2}= & \frac{1}{2} \Delta\left(R_{k j i h} R^{k j i h}\right)-8 \nabla^{k}\left(R^{\jmath p i n}\left(F_{p}^{u} \nabla_{i} F_{u}{ }^{t}\right) R_{k j t h}\right) \\
& -2 H_{k \jmath p i h}^{p} R^{k j i h}-\frac{S}{15}\left(R_{k j i h} R^{k j i h}-\frac{S^{2}}{15}\right) .
\end{aligned}
$$

From (5.25), by taking account of Green's theorem, we have finally the integral formula

$$
\int_{M}\|L\|^{2} d M=-\int_{M}\left(2 H^{p}{ }_{k j p i h} R^{k j i h}+\frac{S}{15}\left(R_{k j i h} R^{k j i h}-\frac{S^{2}}{15}\right)\right) d M,
$$

where $d M$ denotes the volume element of $(M, F, g)$.

Proof of Theorem 5.1. If $(M, F, g)$ satisfies the inequality

$$
2 H_{k j p i n}^{p} R^{k j 2 h}+\frac{S}{15}\left(R_{k j i n} R^{k j i n}-\frac{S^{2}}{15}\right) \geqq 0,
$$

then, we see from (5.26) that $L_{p k j i h}=0$ on $M$, and hence, the tensor field $T$ satisfies the condition (A) in Theorem 3.3. Consequently, from Lemma 4.3 and Theorem 3.3, we have Theorem 5.1.

Q.E.D. 
By direct computation, we have

$$
\left(R_{k \jmath i h}-\frac{S}{30}\left(g_{\jmath i} g_{k h}-g_{k \imath} g_{j h}\right)\right)\left(R^{k j i h}-\frac{S}{30}\left(g^{j i} g^{k h}-g^{k \imath} g^{j h}\right)\right)=R_{k \jmath i n} R^{k \jmath \imath h}-\frac{S^{2}}{15} .
$$

Thus, from (5.26) and the above identity, if a 6-dimensional complete and nonKaehlerian $K$-space is simply connected and satisfies the condition $\left(^{*}\right)$, then it is isometric with a 6 -dimensional sphere $S^{6}$, in which the tensor field $T$ defined by (4.12) satisfies the conditions (A), (B) and (C) in Theorem 3.3. However, we have other examples of $K$-spaces in which the tensor field $T$ defined by (4.12) satisfies the conditions (A), (B) and (C) in Theorem 3.3. The rest of this section will be devoted to show the fact. Let $M=G / K$ be a compact homogeneous space with $\chi(M) \neq 0$, where $G$ is a compact simple Lie group acting effectively on $M$ and $K$ is the isotropy subgroup at some point $x \in M$. Then, $\chi(M) \neq 0$ implies $\operatorname{rank} G=\operatorname{rank} K$. We now assume that the Riemannian metric $g$ of $M$ is determined by a biinvariant metric \langle\rangle on $G$. Furthermore, we assume that $G$ admits an automorphism $\theta$ of order 3 and $K$ is the fixed point set of $\theta$. Since $\operatorname{rank} G=\operatorname{rank} K, \theta$ is an inner automorphism (cf. [6], [24]). Then $M=G / K$ is a reductive homogeneous space. Let $g=\mathfrak{l}+\mathfrak{n}$ be the corresponding orthogonal direct sum decomposition of $\mathfrak{g}$, where $g$ and $f$ are the Lie algebras of $G$ and $K$ respectively. The subspace $\mathfrak{m}$ can be naturally identified with the tangent space at $x=K \in G / K$. Let $\Theta$ be the automorphism of $\mathfrak{g}$ determined by $\theta$. And we put $\left.\Theta\right|_{\mathrm{m}}=-\frac{1}{2} I+\frac{\sqrt{3}}{2} F$, where $I$ denotes the identity. Then $F$ : $\mathfrak{m} \rightarrow \mathfrak{m}$ gives rise to a $G$-invariant almost complex structure (also denoted by $F$ ) on $M$. This almost complex structure $F$ is called the canonical almost complex structure determined by $\theta$. The almost complex structure $F$ satisfies

and

$$
\langle F X, F Y\rangle=\langle X, Y\rangle
$$

$$
[F X, Y]_{\mathfrak{m}}=-F[X, Y]_{\mathfrak{m}}, \quad[X, Y]_{\mathfrak{t}}=[F X, F Y]_{\mathfrak{x}},
$$

for $X, Y \in \mathfrak{m}$ (cf. [5], [24]). On the other hand, since $(G / K, g)$ is a homogeneous Riemannian manifold, the Riemannian connection $\nabla$ of $(G / K, g)$ is given at $x=K$ by the formula:

$$
2\left\langle\nabla_{X} Y, Z\right\rangle=-\left\langle X,[Y, Z]_{\mathfrak{m}}\right\rangle-\left\langle Y,[X, Z]_{\mathfrak{m}}\right\rangle+\left\langle Z,[X, Y]_{\mathfrak{m}}\right\rangle,
$$

for $X, Y, Z \in \mathfrak{m}$. Since $\langle$, is biinvariant, $(G / K, g)$ is naturally reductive with respect to the decomposition $\mathrm{g}=\mathrm{f}+\mathfrak{m}$, i. e., $\left\langle X,[Y, Z]_{\mathfrak{m}}\right\rangle+\left\langle[Y, X]_{\mathfrak{m}}, Z\right\rangle=0$ holds for $X, Y, Z \in \mathfrak{m}$, and furthermore, because of (5.28),

$$
\nabla_{X} Y=\frac{1}{2}[X, Y]_{\mathfrak{m}}, \quad \text { for } \quad X, Y \in \mathfrak{m},
$$

holds at $x=K$. Taking account of (5.27) and (5.29), we have at $x=K$

$$
\left(\nabla_{X} F\right) Y=\nabla_{X}(F Y)-F\left(\nabla_{X} Y\right)
$$




$$
\begin{aligned}
& =\frac{1}{2}[X, F Y]_{\mathfrak{m}}-\frac{1}{2} F[X, Y]_{\mathfrak{m}} \\
& =-F[X, Y]_{\mathfrak{m}},
\end{aligned}
$$

for $X, Y \in \mathfrak{m}$. Thus, $(G / K, F, g)$ is a $K$-space (cf. [5], [24]). It is well known that the curvature tensor $R$ of $(G / K, g)$ is given at $x=K$ by the formula (cf. $[8])$ :

$$
\begin{aligned}
R(X, Y) Z= & -\left[[X, Y]_{\mathfrak{t}}, Z\right]-\frac{1}{2}\left[[X, Y]_{\mathfrak{m}}, Z\right]_{\mathfrak{m}} \\
& +\frac{1}{4}\left[X,[Y, Z]_{\mathfrak{m}}\right]_{\mathfrak{m}}-\frac{1}{4}\left[Y,[X, Z]_{\mathfrak{m}}\right]_{\mathfrak{m}}, \quad \text { for } \quad X, Y, Z \in \mathfrak{m} .
\end{aligned}
$$

Now, let $T$ be a $G$-invariant tensor field of type $(1,2)$ on $M=G / K$ determined by

$$
T(X, Y)=\frac{1}{2}[X, Y]_{\mathrm{m}}, \quad \text { for } \quad X, Y \in \mathfrak{m} .
$$

Since $(G / K, g)$ is naturally reductive, $T$ is skew-symmetric. Taking account of (5.30), we obtain at $x=K$

$$
\begin{aligned}
T(X, Y) & =\frac{1}{2}[X, Y]_{\mathfrak{m}} \\
& =-\frac{1}{2} F\left(F[X, Y]_{\mathfrak{m}}\right) \\
& =\frac{1}{2} F\left(\nabla_{X} F\right) Y, \quad \text { for } \quad X, Y \in \mathfrak{m} .
\end{aligned}
$$

Thus, the tensor field $T$ coincides with the tensor field given by (4.12) and hence, satisfies by Lemma 4.3 the conditions (B) and (C) in Theorem 3.3. Furthermore, taking account of (5.29), (5.31) and (5.32), we can easily show that $T$ satisfies the condition (A) in Theorem 3.3. The following spaces are examples of 6-dimensional compact, simply connected and non-Kaehlerian $K$-spaces (cf. $[5],[24])$ :

$$
S U(3) / S(U(1) \times U(1) \times U(1)), \quad S O(5) / U(2), \quad G_{2} / S U(3)=S^{6} .
$$

\section{$\S 6$. Some 4-dimensional $F$-spaces and $H$-spaces.}

In [12], Sawaki and the present author proved that any $F$-space with nonvanishing pointwise constant holomorphic sectional curvature is a Kaehlerian space. On the other hand, in [21], Tricerri and Vanhecke gave an example of non-Kaehlerian locally flat almost Hermitian manifolds of dimension 4 . Obviously, any locally flat almost Hermitian manifold is an $F$-space. Therefore, it might be interesting to give some sufficient conditions for an almost Hermitian manifold to be Kaehlerian in terms of the curvature tensor and others. In connection with this, we shall prove the following 
THEOREM 6.1. Let $(M, F, g)$ be a 4-dimensional H-space satısfying the condition (*). If the scalar curvature $S$ of $(M, F, g)$ is non-negative on $M$, then $(M, F, g)$ is a Kaehlerian space.

Taking account of Theorem 3.1, Theorem 6.1 follows immediately from the following

LEMMA 6.2. Let $(M, F, g)$ be a 4-dimensional almost Hermitian manifold satisfying the condition $\left(^{*}\right)$. If the scalar curvature $S$ of $(M, F, g)$ is non-negatwe on $M$, then $S \geqq S^{*}$ holds on $M$.

Proof. We now put $B_{k \jmath i h}=g\left(R\left(e_{k}, e_{\jmath}\right) e_{\imath}, e_{h}\right), 1 \leqq k, \jmath, \imath, h \leqq 4$, for an orthonormal basis $\left(e_{2}\right)=\left(e_{1}, e_{2}, e_{3}, e_{4}\right)$ in $T_{x}(M)$ at each point $x \in M$. Taking account of the arguments developed in [15], at each point $x \in M$, we may choose an orthonormal basis $\left(e_{2}\right)$ in $T_{x}(M)$ in such a way that one of the following conditions ( I )-(i), ( I )-(ii), ( I )-(ii)', ( I )-(iii), (II), (III), (IV) and (V) holds :

( I )-(i) $\quad B_{1212}=B_{1313}=B_{1414}=B_{2323}=B_{2124}=B_{3434}=-\lambda / 3 \quad(\lambda \neq 0)$,

otherwise, $B_{k j i n}$ being zero, and $4 \lambda=S$;

( I )-(ii)

$$
\begin{aligned}
& B_{1212}=B_{3434}=-2 \lambda / 3, \quad B_{1313}=B_{2424}=B_{1414}=B_{2323}=-\lambda / 6, \\
& B_{1234}=\lambda / 3, \quad B_{1423}=-\lambda / 6, \quad B_{1342}=-\lambda / 6 \quad(\lambda \neq 0),
\end{aligned}
$$

otherwise, $B_{k j \imath h}$ being zero, and $4 \lambda=S$;

$$
\begin{array}{cl}
\text { ( I )-(ii) }{ }^{\prime} & B_{1212}=B_{3434}=-2 \lambda / 3, \quad B_{1313}=B_{2424}=B_{1414}=B_{2323}=-\lambda / 6, \\
& B_{1234}=-\lambda / 3, \quad B_{1423}=\lambda / 6, \quad B_{1342}=\lambda / 6 \quad(\lambda \neq 0),
\end{array}
$$

otherwise, $B_{k j i n}$ being zero, and $4 \lambda=S$;

( I )-(iii) $\quad B_{1212}=B_{3434}=-\lambda \quad(\lambda \neq 0)$,

otherwise, $B_{k j i n}$ being zero, and $4 \lambda=S$;

$$
B_{1212}=-\lambda, \quad B_{3434}=-\mu \quad(\lambda \neq \mu, \lambda, \mu \neq 0),
$$

otherwise, $B_{k j i n}$ being zero, and $2(\lambda+\mu)=S$;

$$
B_{1212}=B_{1313}=B_{2323}=-\lambda / 2 \quad(\lambda \neq 0),
$$

otherwise, $B_{k j \imath h}$ being zero, and $3 \lambda=S$;

$$
B_{1212}=-\lambda \quad(\lambda \neq 0),
$$

otherwise, $B_{k j i n}$ being zero, and $2 \lambda=S$;

$$
B_{k j i h}=0, \quad 1 \leqq k, j, i, h \leqq 4 \text {. }
$$


We now put $f_{j i}=g\left(F e_{\jmath}, e_{\imath}\right), 1 \leqq \jmath, \imath \leqq 4$. Then, taking account of $g\left(F e_{\jmath}, F e_{\imath}\right)=$ $g\left(e_{\jmath}, e_{\imath}\right)=\delta_{\jmath i}$, we have

$$
\left(f_{j i}\right)=\left(\begin{array}{cccc}
0 & f_{12} & f_{13} & f_{14} \\
-f_{12} & 0 & -f_{14} & f_{13} \\
-f_{13} & f_{14} & 0 & -f_{12} \\
-f_{14} & -f_{13} & f_{12} & 0
\end{array}\right) \text {, }
$$

or

$$
\left(f_{j 2}\right)=\left(\begin{array}{cccc}
0 & f_{12} & f_{13} & f_{14} \\
-f_{12} & 0 & f_{14} & -f_{13} \\
-f_{13} & -f_{14} & 0 & f_{12} \\
-f_{14} & f_{13} & -f_{12} & 0
\end{array}\right),
$$

where $\left(f_{12}\right)^{2}+\left(f_{13}\right)^{2}+\left(f_{14}\right)^{2}=1$.

We here consider eight cases $(\mathrm{A}) \sim(\mathrm{H})$ as followings. By the definition of $S^{*}$, we have

$$
-2 S *=\sum_{a, b, \jmath, k} f_{b a} B_{b a k \jmath} f_{k \jmath} .
$$

(A) In the case ( I )-(ii), we have from (6.3)

$$
\begin{aligned}
S^{*}= & -2\left(f_{12} B_{1212} f_{12}+f_{12} B_{1234} f_{34}+f_{13} B_{1313} f_{13}+f_{13} B_{1324} f_{24}\right. \\
& +f_{14} B_{1414} f_{14}+f_{14} B_{1423} f_{23}+f_{23} B_{2314} f_{14}+f_{23} B_{2323} f_{23} \\
& \left.+f_{24} B_{2413} f_{13}+f_{24} B_{2424} f_{24}+f_{34} B_{3412} f_{12}+f_{34} B_{3434} f_{34}\right) \\
= & -(2 \lambda / 3)\left(2 f_{12} f_{34}+f_{13} f_{24}-f_{14} f_{23}-4\left(f_{12}\right)^{2}-\left(f_{13}\right)^{2}-\left(f_{14}\right)^{2}\right) .
\end{aligned}
$$

Thus, when $\left(f_{j \imath}\right)$ has the form (6.1), we have

and hence

$$
S^{*}=4 \lambda\left(f_{12}\right)^{2},
$$

$$
S *=S\left(f_{12}\right)^{2} .
$$

When $\left(f_{\jmath \imath}\right)$ has the form (6.2), we have

and hence

$$
S^{*}=4 \lambda / 3,
$$

$$
S^{*}=S / 3 \text {. }
$$

(B) In the case (I )-(ii)', we have similarly

$$
S^{*}=\stackrel{=}{=} / 3 \text {, }
$$


when $\left(f_{j i}\right)$ has the form (6.1), and

$$
S^{*}=S\left(f_{12}\right)^{2},
$$

when $\left(f_{j i}\right)$ has the form (6.2).

(C) In the case (I)-(i), we have

$$
\begin{aligned}
S^{*} & =(2 \lambda / 3)\left(\left(\int_{12}\right)^{2}+\left(f_{13}\right)^{2}+\left(f_{14}\right)^{2}+\left(f_{23}\right)^{2}+\left(f_{24}\right)^{2}+\left(f_{34}\right)^{2}\right) \\
& =4 \lambda / 3=S / 3 .
\end{aligned}
$$

(D) In the case (I )-(iii), we have

$$
\begin{aligned}
S^{*} & =(2 \lambda)\left(\left(f_{12}\right)^{2}+\left(f_{34}\right)^{2}\right)=(4 \lambda)\left(f_{12}\right)^{2} \\
& =S\left(f_{12}\right)^{2} .
\end{aligned}
$$

(E) In the case (II), we have

$$
\begin{aligned}
S^{*} & =2\left(\lambda\left(f_{12}\right)^{2}+\mu\left(f_{34}\right)^{2}\right) \\
& =S\left(f_{12}\right)^{2}
\end{aligned}
$$

(F) In the case (III), we have

$$
S^{*}=\lambda=S / 3 \text {. }
$$

(G) In the case (IV), we have

$$
S^{*}=2 \lambda\left(f_{12}\right)^{2}=S\left(f_{12}\right)^{2} .
$$

(H) In the case ( V), it is evident that

$$
S^{*}=S=0 \text {. }
$$

Taking account of $(6.4) \sim(6.13)$, we can prove Lemma 6.2.

Q.E.D.

Taking account of the arguments developed in the proof of Lemma 6.2, we can prove the following Propositions 6.3 and 6.4.

Proposition 6.3. Let $\left(M, F_{0}, g_{0}\right)$ be a Kaehlerian space of constant holomorphic sectional curvature $c(>0)$ of complex dimension 2 and $F$ be an almost complex structure on $M$ such that $\left(M, F, g_{0}\right)$ is an $H$-space. Then, $F=F_{0}$ or $F=-F_{0}$.

Proof. From the hypothesis for $\left(M, F_{0}, g_{0}\right)$, at each point $x \in M$, only the case (I )-(ii)' with $\lambda=3 c / 2$ occurs with respect to any orthonormal basis $\left(e_{2}\right)=$ $\left(e_{1}, e_{2}=F_{0} e_{1}, e_{3}, e_{4}=F_{0} e_{3}\right)$ in $T_{x}(M)$. Since the scalar curvature of $\left(M, F, g_{0}\right)$ is positive and constant on $M$, from Theorem $6.1,\left(F, g_{0}\right)$ is a Kaehlerian structure on $M$. Thus, from Theorem 3.1, and (6.6), (6.7), we have finally 
or

$$
F e_{1}=e_{2}=F_{0} e_{1}, \quad F e_{3}=e_{4}=F_{0} e_{3},
$$

$$
F e_{1}=-e_{2}=-F_{0} e_{1}, \quad F e_{3}=-e_{4}=-F_{0} e_{3} \text { Q. E. D. }
$$

Proposition 6.4. Let $\left(M, F_{0}, g_{0}\right)$ be a Kaehlerian space of constant holomorphic sectional curvature $c(\neq 0)$ and $F$ be an almost complex structure on $M$ such that $\left(M, F, g_{0}\right)$ is an $F$-space. Then, $F=F_{0}$ or $F=-F_{0}$.

Proof. In general, in an $F$-space $(M, F, g)$, from (3.3) and (3.4), it follows that $S=S^{*}$. Thus, taking account of (6.6) and (6.7), we have finally

or

$$
\begin{aligned}
& F e_{1}=e_{2}=F_{0} e_{1}, \quad F e_{3}=e_{4}=F_{0} e_{3}, \\
& F e_{1}=-e_{2}=-F_{0} e_{1}, \quad F e_{3}=-e_{4}=-F_{0} e_{3} . \quad \text { Q. E. D. }
\end{aligned}
$$

\section{REFERENCES}

[1] W. Ambrose And I. M. Singer, On homogeneous Riemannian manifolds, Duke Math. J., 25 (1958), 647-670.

[2] J.E. D'atri and H.K. Nickerson, Geodesic symmetries in spaces with special curvature tensors, J. Diff. Geom., 9 (1970), 251-262.

[3] S. FujImURA, A remark on Lichnerowicz's theorem for locally symmetric Riemannian manifolds, Memoirs of the Research Institute of Science and Engineering, Ritsumeikan Univ., 27 (1974), 1-3.

[4] T. Fukami and S. Ishihara, Almost Hermitian structure on $S^{6}$, Tōhoku Math. J., 7 (1955), 151-156.

[5] A. Gray, Riemannian manifolds with geodesic symmetries of order 3, J. Diff. Geom., 7 (1972), 343-369.

[6] A. GRAY, Kähler submanifolds of homogeneous almost Hermitian manifolds, Tōhoku Math. J., 21 (1969), 190-194.

[7] S. Kobayashi, Transformation groups in Differential Geometry, Springer-Verlag, Berlin, Heidelberg, New York, 1972.

[8] S. Kobayashi and K. Nomizu, Foundations of Differential Geometry, I, II, Interscience Publishers, New York, 1963, 1969.

[9] M. Matsumoto, On 6-dimensional almost Tachibana spaces, Tensor N.S., 23 (1972), 250-252.

[10] K. Nomizu and K. Yano, Some results to the equivalent problem in Riemannian geometry, Proc. United States-Japan Sem. in Kyoto, Japan, 1965, 95-100.

[11] Y. OGAWA, A condition for a compact Kaehlerian space to be locally symmetric, to appear.

[12] S. Sawaki and K. Sekigawa, Almost Hermitian manifolds with constant holomorphic sectional curvature, J. Diff. Geom., 9 (1974), 123-143.

[13] K. Sekigawa, Some hypersurfaces satisfying $R(X, Y) \cdot R=0$, Tensor N. S., 25 (1972), 133-136.

[14] K. SeKigaiva, On some 3-dimensional complete Riemannian manifolds satisfying $R(X, Y) \cdot R=0$, Tōhoku Math. J., 27 (1975), 561-568.

[15] K. SEKIGAWA, On some 4-dimensional Riemannian manifolds satisfying $R(X, Y)$ $\cdot R=0$, Hokkaido Math, J., 2 (1977), 216-229. 
[16] K. Sekigawa, Notes on homogeneous almost Hermitian manifolds, to appear in Hokkaido Math. J..

[17] K. Sekigawa and H. TAKagi, On conformally flat spaces satisfying a certain condition on the Ricci tensor, Tōhoku Math. J., 23 (1971), 1-11.

[18] I. M. Singer, Infinitesimally homogeneous spaces, Comm. Pure Appl. Math., 13 (1960), 685-697.

[19] H. TAKAGI, An example of Riemannian manifolds satisfying $R(X, Y) \cdot R=0$, but not $\nabla R=0$, Tōhoku Math. J., 24 (1972), 105-108.

[20] K. Takamatsu, Some properties of 6-dimensional $K$-spaces, Kōdai Math. Sem. Rep., 23 (1971), 215-232.

[21] F. TRICERri and L. Vanhecke, Flat almost Hermitian manifolds which are not Kähler manifolds, Tensor N.S., 31 (1977), 249-254.

[22] E. TsukADA, Transitive actions of compact connected Lie groups on symmetric spaces, Sci. Rep. Niigata Univ., 15 (1978), 1-19.

[23] Watanabe and K. Takamatsu, On a $K$-space of constant holomorphic sectional curvature, Kōdai Math. Sem. Rep., 25 (1973), 297-306.

[24] J. Wolf and A. Gray, Homogeneous spaces defined by Lie group automorphisms, I, II, J. Diff. Geom., 2 (1968), 77-114, 115-159.

[25] S. Yamaguchi, G. Chüman and M. Matsumoto, On a special almost Tach1bana space, Tensor, N.S., 24 (1972), 351-354.

[26] K. Yano, Differential geometry on complex and almost complex spaces, Pergamon Press, 1965. 